\title{
ENHANCED TELEVISION AND INTERACTIVE SERVICES
}

Claire Dormann

Centre for Tele-Information, Technical University of Denmark.

Key words: interactive television, enhanced television, tele-commerce, entertainment.

\begin{abstract}
Interactive Television (ITV) is a recent and key development in the new media landscape with new services being launched in many countries. Thus to understand this technology it is useful to review its recent development. This paper is based on the state of the art in interactive television. The state of the art will serve as the basis for discussion on the future development of enhanced television. We will start by expanding on current applications then present issues that might be fundamental for the design of interactive television.
\end{abstract}

\section{INTRODUCTION}

Interactive television (ITV) is rapidly expanding, as many consumers have access to television, ITV could become a key development in the information or perhaps in the infotainment society. As we are in the early stages of such a development, critical knowledge can be gained by reviewing current applications and trying to determine factors that could prove decisive for the design of future services.

This paper summarises the current composition of the ITV landscape by a the state of the art look at interactive programmes and services. Our survey of the state of the art serves two purposes: it provides information about this new technology and serves as the starting point for discussion of issues related to the design of enhanced television.

In this paper, we will present an overview of ITV. We see three aspects in ITV: the electronic programme guide or portal (the navigation system), 
enhanced television (including TV and interactive programmes) and interactive services (stand alone applications). Thus we will first review interactive services and then introduce interactive programmes in a more detailed manner, as the emphasis of this paper is on enhanced television.

To complete this study, we will build on existing interactive programmes to suggest development in new areas of enhanced television. For example coupling chat and talk shows could enhance the value of these television (TV) programmes and perhaps lead to new forms of entertainment.

However, to support long term development, we will need to find criteria which will ensure the success of such applications and distinguish this media from its predecessors. Therefore, we have turned to television to study programme characteristics that might prove essential to the design of enhanced television. As television is related to entertainment: emotions, narratives and intimacy might become crucial parameters for future developments.

\section{INTERACTIVE TELEVISION}

In order to introduce recent developments in ITV, an overview of different interactive services and programmes is first presented. This overview is mainly based on a review of 'on-air' ITV providers as we have found very few papers dedicated to interactive television services $[1,2,3]$. Carey [1] and Steward's [2] papers provide useful insights for this study by describing earlier trials. Taking a more business oriented approach, Crowne [3] provides a short overview of the current ITV spectrum dividing applications between, content driven, value added and enhanced television. The category added-value (telebanking and education) was found problematic as it is thought that most ITV applications bring added value (see 5.3).

This overview is thus organised according to a taxonomy of television genres which has proved useful for highlighting differences and trends in interactive television. Different genres of television have often been contrasted such as, entertainment and information. TV programmes can be situated in this triad: Information, Play (or entertainment) and Fiction [4]. It should be noted that no evidence of interactive programming has been found in relation to fiction, however we are expecting this only to be a question of time.

Quite a large number of ITV applications can be placed in the entertainment pole (related to interactive games, game shows, or interactive magazines). There are very few applications related to the information pole (interactive weather and dictionary), although demos for wild life and pop 
programmes have been mentioned [5]. Chat and email can be seen as information or entertainment, however in the home context, we expect their main usage to be a form of entertainment. Due to the pervasive nature of commerce in interactive television, we have added this dimension to the television triad to cover many interactive applications. Telecommerce includes teleshopping, interactive advertising, and home shopping. We can qualify ITV applications as situated in Figure 1, TV being in the centre of the diamond-shape.

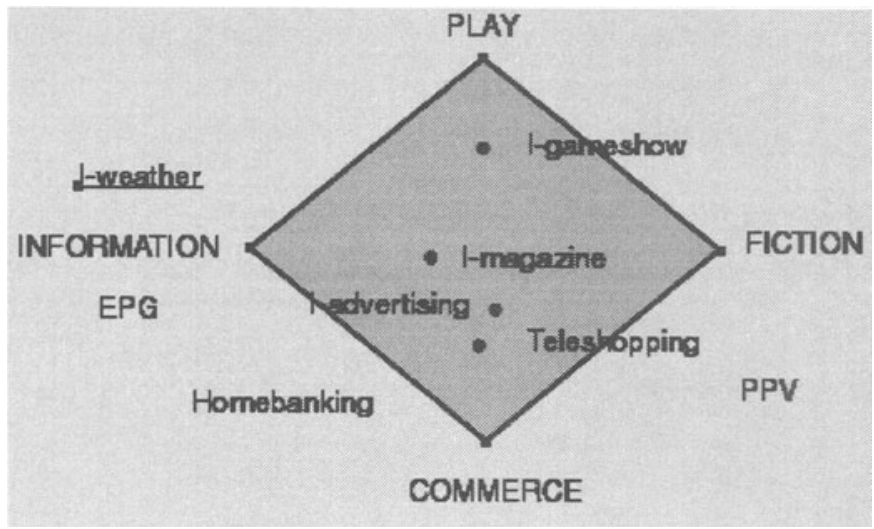

Figure 1. Interactive television

Most interactive programmes and services have a relation with more than one pole. For example home banking has an element of information (finance) and commerce (bank), and interactive ads can have an element of information (product) and play (game).

\section{INTERACTIVE SERVICES}

Electronic programme guides (EPGs) can be seen as the entrance to television programmes as well as interactive services. These services consist mainly of home shopping services or entertainment services. The weather service is the only type of service which does not fit this category.

EPGs help people grasp a planning concept, understand complex programmes and services, and navigate in the ITV environment [6]. In home shopping, consumers are able to buy a range of products such as toys, groceries, CD and books, or to plan and order trips and tickets [7]. Another popular application is home banking including account management and financial services. Other interesting services are small ads for homes' rental 
and job offers. Pay Per View (PPV) can also be considered as a form of home shopping. PPV is a widespread service where viewers can select and pay for video on demand (or nearly on demand) specific live-sport events or spectacles. In the future consumers might also have access to news or games on demand.

Entertainment services include interactive games consisting of small computer or family games integrated into an arcade game. Network games are also appearing: teleplayers will be able to compare scores or compete against each other. Interactive spectacle magazines can also be provided, giving information about film, music and theatre (integration with a booking facility is also planned).

Chat applications are also available, enabling users to exchange textbased messages, allowing easy and efficient discussions. The chat users will also be able to connect to similar services on the internet. Electronic mail and more rarely Word Wide Web (web) access are also possible [8].

\section{INTERACTIVE PROGRAMMING}

Interactive programmes are initiated from TV programmes, the most frequent examples are situated in telecommerce with interactive advertising and teleshopping. We are going to review these applications in detail.

\subsection{Interactive advertising}

Today, interactive advertising (ad) is synchronised with the TV ad. An interactive overlay or icon is generated on the screen leading to the interactive component. When specific screens are accessed, viewers can learn more about products but generally, other forms of interactions are also proposed.

Viewers can order catalogues, can take a product test, participate in competitions, draw, or play games. Examples of products featured interactive ads include cars and holiday breaks.

An interactive ad should be brief in order not to interfere with the programme that viewers wish to watch. The message must be simple and quick. This strategy is based on provoking an impulsive response (look at the interactive ad) resulting in the required action (ordering the catalogue). However this limits the creative possibilities of the ads. A natural extension of this concept would be to enable consumers to order directly.

Questions under discussion concern the impact of this kind of advertising on other TV ads or the impact of the interactive ad on attitude toward products, or purchasing behaviour (especially as the novelty effect wears 
off). Moreover interactivity might demand that ad messages are reformulated, this is essential for talking differently about a product. It is necessary to create a complicity with consumers so that they can appropriate the brand. The communication must adapt and re-invent itself. Besides, integrating TV and multimedia will lead to marketing of mass customisation.

\section{$4.2 \quad$ Teleshopping}

Teleshopping usually takes the form of a talk show presented by hosts interacting with an audience (guests, studio audience and outside consumers). The objectives of such programmes are to give viewers the feeling of trying products. Viewers assist in the contextualisation of products. The products' merits are demonstrated in every dimension allowed by the medium. In some ways we can consider teleshopping the multimedia counterpart missing from web shops. Consumers can now see the product in use, be enticed by attractive features, and seductive plots and thus be tempted to buy.

In interactive teleshopping consumers can directly order products shown in the programme. During the show, an icon appears signalling to viewers that they can now buy an item. The chosen product is then automatically displayed. Viewers enter the quantity and pay by inserting their credit card in the set-up box card reader [9] thus making telecommerce more secure than electronic commerce.

\subsection{Play: interactive TV games}

Interactive games take place in relation to game shows, allowing viewers to participate in the game. It has developed for a large spectrum of TV shows ranging from sports to cooking [10]. Instances of interactive betting for horse racing have also been suggested.

\subsection{Encyclopaedic information}

Another kind of interactive feature, an information service, is a dictionary (the Dico) added to a programme to facilitate the acquisition and comprehension of information [11]. Numerous documentaries could benefit from the addition of an informative interactive module. A feature like the Dico could be specific to a particular programme or become a permanent feature available with a range of programmes. 


\subsection{Sequencing of interactive programmes}

Most interactive programmes occur concurrently with TV programmes. Viewers play at the same time as a game show is running, viewers read dictionary definitions while watching a programme, and they can buy, or place a bet, while the show continues in the background. By contrast interactive ads imply a disruption of the TV ad, however brief. The viewers cannot watch the programme and interact with the ad at the same time.

\section{SCENARIOS FOR INTERACTIVE PROGRAMMES}

We are only at the beginning of interactive television, thus we have only found a few examples of interactive programmes however, more should be coming in the next few months. To accustom TV viewers to interactivity, these programmes should remain simple. Exploring broadcaster web sites lets us discover how a whole range of interactive programmes could be generated. It is envisaged that some forms of interactive programmes (infosheet) could prove better suited to specific TV genres while others such as quizzes, chat forums, and shops could prove pervasive to most TV programmes.

\subsection{Infosheet}

A quick look at CNN [12] suggests different possibilities for interactive news programmes such as news of the day, contextual information, or transcripts of interviews. News of the day could consist of news headlines. Contextual information, for the flood in Venezuela, could be given in the form of maps, and statistical information thus helping viewers to situate or understand the impact of the event.

Similarly, for BBC food and drink programme, recipes and instructions could be added to the show as an interactive element. Alternatively the recipe (as a few lines of text) could also be shown concurrently to the TV programme. More complex information could include cooking tips of the type "would you like to know the best way to peel a garlic clove? we've got 60 useful tips to share." [13]. 


\subsection{Take a quiz, chat and shop}

We have found numerous instances of quizzes in web sites, from news programmes $(\mathrm{CNN})$, language programme (TV5), StarTrek (BBC), as well in ITV (interactive advertising). A quiz could be taken after virtually any kind of TV programmes.

TV5 is an international French speaking channel which offers language programmes. In the TV5 web site [14], users can take the exercise of the week. In the same way, such tests could also be taken after the TV programme. Alternatively, some questions could be added while the programme is running.

In electronic commerce, chat rooms are used by consumers exchanging tips and information and for special events such as authors discussing books on-line. All kinds of scenarios are possible with interactive programmes.

For a cultural TV programme like 'Bouillon de culture' where authors, or directors, are invited to present their works, the TV programme could be enhanced by having guests answering questions from the TV audience in a chat room. Debates could be organised after virtually any programmes. Chat rooms are an example of a social community building up around a TV programme with conversations linking in and out of the broadcast material.

It is always possible to sell something related to a TV programme such as TV goods with series (Fans of StarTrek, for you, your own special video). If viewers are watching a travel show being presented, holiday booking might seem highly desirable. A viewer could be directed to a specific screen like 'My vacation in Crete' or to an interactive service like a travel agency. Music channels and programmes seem prime candidates for teleshopping, as compact disks are some of the most common items sold through the internet.

What remains to be seen is to what degree TV viewers would accept being subjected to commercial offers. What might be acceptable for the travel show might become intrusive for a news programme.

\subsection{Added Value}

Interactive programmes often occur as a natural extension of the TV genre, enhancing the primary characteristics of the programme (play for game shows, or purchases for teleshopping). We have suggested similar developments (chat and talk shows, or information and news) although as interactive programmes evolve new forms might emerge.

Interactive programming enhances the TV viewing experience. By allowing each viewer to participate in the game, we reinforce the experiential quality of the game show programme, thus boosting the audience interest and engagement. If viewers can interact and communicate 
with TV personalities and hosts, the emotional impact of the programme could be reinforced. We allow TV viewers to become active participants.

Interactive programming can also work by adding layers of depth to the TV programme. This might be by adding extra information (the Dico) or functionality (shopping). By bringing complementary information, the comprehension and assimilation of the TV information is facilitated. Ordering a catalogue after an advertisement, or buying from teleshopping seems a natural extension of the function of these programmes.

Moreover we are rewarding the TV audience, by giving away discount (interactive ad), by providing supplementary facilities (shopping) and by allowing viewer participation.

\section{ENHANCED TELEVISION}

So far, we have suggested, very simple examples of interactive programmes as being well suited to this new medium. For more complex forms of interactive programming, we have to understand TV characteristics and TV consumption as it might indicate phenomena that might prove fundamental to the design of enhanced television. In doing so we might highlight fundamental differences between web sites and interactive programmes.

\subsection{Home context and ITV}

An important aspect of ITV is the home context which might influence the utilisation and design of future applications. The home space is often described in different terms than the office context and computer world. It is highlighted by a Phillips project on smart homes which described the living room (often containing the main TV set) in the following terms, a communal family space where many activities are taking place such as relaxation, communication and entertainment [15]. These are also the primary motives for watching television. which contrasts it, from the internet defined in terms of rationality, facts and tasks [16].

It might also prove an important element of ITV. For example every interactive programme and service might need to contain an element of entertainment and communication. 


\subsection{Television and emotions}

Television is not the world wide web, it is about entertainment, emotions and being really compelling [17]. Emotions are at the heart of TV programmes. This is shown by looking more closely at the rhetorical intent of TV programmes: provoke beliefs and adhesion in political programmes, provoke laughter in comic programmes, provoke tears in reality shows and magazines, or provoke lust in adult programmes. Even information programmes such as news are often emotive and spectacular, by showing slow motion sequences of horrendous accidents or scenes of devastation from multiples angles [18].

It is important to remember that all TV programmes shared these characteristics: emotions, pleasures and spectacles. It is thought that these criteria should also play an important part in enhanced television. We expect ITV to rely more than their web counterpart on visual persuasive techniques that make programmes visually appealing and have an emotional impact on viewers.

\subsection{Parasocial aspect of television}

A key to television, especially in relation to the web, is that television brings other people into our homes. Viewers, especially in some shows have ample opportunity to form parasocial relationships -feeling of friendships or intimacy on the viewers part with remote 'personae' such as soap opera characters, news and talk-show hosts [19]. Television personalities, encourage parasocial involvement by using a conversational style and gestures. Hosts embed related comments in personal revelations and questions directed to viewers, in an effort to create positive feeling,

We should also stress the importance of social relation in TV consumption. People engage in social viewing for example while watching video or football matches. Many discussions are also generated around TV programmes.

This may have important consequences for the design of interactive television. These services may take a more personal and human point of view, for example by using hosts and celebrities in telecommerce or by giving a more intimate and subjective touch to interactive programmes. Moreover, people might want to interact with enhanced TV in groups rather than on an individual basis, play game as a 'unit' against relatives and friends rather than anonymous teleplayers. 


\subsection{Toward an enhanced television model}

As we have seen, characteristics such as intimacy, emotions, and entertainment might play a fundamental role in ITV, not only for enhanced TV, but also for interactive services. Moreover TV is a dynamic linear, medium, thus we anticipate a heavier reliance in ITV on film and game narratives based on affective and engaging plots. This could distinguish ITV from the more factual approach of web sites.

However, in the future, if enhanced TV should prove compelling, we will need to determine the effects of interactive programmes. Besides affective and parasocial factors, interactivity is expected to play a crucial role.

Interactivity has been described by Jensen [20] in term of registration, transmission, consultation (some selective choice), and conversation (like in chat). Transmission corresponds to a zero degree of interactivity and registration to TV providers collecting information on viewers, thus viewer experience could be described by consultation and conversation. We might need to refine this classification by adding participation to distinguish between passive selection and active participation (e.g games).

Other factors to take into consideration in the evaluation of enhanced television are vividness (the ability of a technology to produce a sensorially rich mediated environment) and attention (increased attention due to participation). According to Hoffman's model for compelling on-line experience, factors such as telepresence, playfulness and challenges should also be fundamental [21]. We should thus integrate these factors and develop a model adapted to enhanced television.

\section{CONCLUSION}

In this paper, we have presented the state of the art of ITV applications describing interactive programmes and services. In doing so, we have highlighted two main trends in the development of these applications: telecommerce and entertainment, centred around the provision of games.

In order to highlight the potential of this new media, we have also suggested new developments such as the addition of chat forums to TV programmes. It is important to remember that one of the main use of the internet at home is communication thus exploiting this possibility might ensure the success of ITV. The success of an experiment in inhabited TV, extending traditional TV broadcast by enabling social interaction among participants through a series of virtual worlds [22] might confirm this trend.

While reviewing interactive programmes we have discussed TV characteristics that might prove fundamental aspects of ITV: social and 
parasocial aspects and emotions. We have also presented factors that should intervene in describing compelling enhanced Television

In the future, we expect more correspondence between interactive programmes and services in telecommerce, or in games. For example viewers could practice playing Mastermind (a form of Trivial Pursuit) by accessing the game from the game arcade, and then participating live with the interactive programme. Furthermore a greater integration and polarisation around the three spheres: entertainment, telecommerce and information could take place. Visionik [23] refers to the notion of interactive entertainment channel (IEC) integrating games, game shows, events and the interactive entertainment magazine.

To a certain degree the attainmrnt of ITV might depend on its successful promotion. Viewers will be persuaded to try these services with appealing portals describing each services, bringing up-to-date developments and, organising competitions or sweeptakes. Television is about entertainment, the success of interactive television will depend on our ability to create an interesting, exciting and fun experience.

\section{ACKNOWLEDGMENT}

This project has been financed by CIT, the Danish national center for IT research, in the framework of the 'Multimedier og hjemmene' project. A special thanks to Cathy Toscan.

\section{REFERENCES}

[1] Carey J. (1997) "Interactive television trials and marketplace experiences", Multimedia tools and applications 5, 207-216.

[2] Crowne A. (1999) "Where's the money in interactive digital services", IBC'99, Amsterdam, 54-60.

[3] Stewart J. (1998) "Interactive television at home: television meets the internet", Human and social factors of ITV, Carat, April 1999, http://www.itvnews.com/research /tvand1.htm

[4] Jost F. (1997) "La promesse des genres", Resaux 81, CNET http://www.cnet.fr/publi/welcome.html

[5] Sims M. (1999) "Television and the internet: from aiming too high to aiming too low", Intermedia 27, 3, 4-6.

[6] Ehrmantraut M. Hårder T. Wittig H. and R. Steinmetz (1996) "The Personnal electronic programme guide- towwards the pre-selection of individual TV programmes”, CIKM'96 Rockville, USA, 243-250

[7] BSkyB consortium http://www.bib.co.uk/, December 1999

[8] WebTV net http://www.webtv.net , November 1999 
[9] Cannal Sattelite http://www.canalsatellite.fr/, September 1999

[10] Two Way TV http://www.twowaytv.com, September 1999

[11] TPS http://www.tps.fr/bienvenue.htm November 1999

[12] CNN http://www.CNN.com/ December 1999

[13] BBC http://www.bbc.co.uk/_December 1999

[14] TV5 http://www.TV5.fr/ December 1999

[15] Smart Homes http://www.philips.com/casa/index.html, July 1999

[16] Potts R., Dedmon A. and Halford J. (1996) "Sensation seeking, television viewing motives and home television viewing patterns", 21, 6 1081-1984.

[17] Saint Girons R. (1999) "Convergence myths and reality -the driving force behind digital interactive TV”, IBC'99, Amsterdam, 391-392

[18] Noel N. (1997) "Genericite, sequentialite, esthetique visuelles", Resaux 81 CNET http://www.cnet.fr/publi/welcome.html

[19] Stephens D. , Hill R. and Bergman K. (1996) "Enhancing the consumer-product relationship: lessons from the QVC home shopping channel", Joumal of business reseach, 37, 193-200

[20] Jensen J. (1999) "The concept of interactivity". In. Jens F Jensen and Cathy Toscan (Eds.), "Interactive television", Aalborg University, Denmark 100-160

[21] Hoffman D. and Novak T. (1995-1998) "Marketing in hypermedia computer environments: conceptual foundation" http://www2000. ogsm.vanderbilt.edu/

[22] Benford C., Walker G. and J. Wyver (1998) "Experiments in ITV", CHI'98, USA, 289290

[23] Visionik BBC http://www.visionik.dk/ November 1999 